\section{Effect of Particle Size Distribution on Column Efficiency in Gel Chromatography $^{\dagger}$}

\author{
Kazuhiro NaKanishi. Shuichi Yamamoto, \\ Ryuichi MATSUNO and \\ Tadashi Kamikubo \\ Department of Food Science and Technology, \\ Faculty of Agriculture, Kyoto \\ University, Kyoto 606 \\ Received April 18, 1978
}

Column efficiency in gel chromatography is influenced by a number of factors such as particle size, flow rate and degree of cross-linkage of gels. Particle size distribution of packing materials is also considered one of the important factors. It has been empirically found that column efficiency is improved by narrowing the particle size distribution. ${ }^{\text {s) }}$ However, there have been reported only a few studies about this particle size distribution effect other than that of de Vries et al. ${ }^{4}$ In this work, we examined the effect of particle size distribution on column efficiency, both experimentally and theoretically. Particularly, it was studied what mean diameter should be used as representative size when the particle size distribution is broad. Dextran crosslinked gels, i.e., Sephadex G-25 Medium, G-25 Coarse, G-150 and their sieve fractions were used as packing materials.

According to the dispersion model, ${ }^{1,5 \sim 7)}$ chromatography proceeds in the stationary phase (gel phase) and the mobile phase outside gel particles. In a column packed with gels having diameters represented by distribution function $f\left(D_{p}\right)$, i.e., $\int_{0}^{\infty} f\left(D_{p}\right) d D_{p}=1$, the mass balance equations of a solute in the mobile phase and in the gel phase are expressed by Eqs. (1) and (2), respectively.

$$
\begin{gathered}
\frac{\partial C}{\partial t}=D_{l} \frac{\partial^{2} C}{\partial z^{2}}-u \frac{\partial C}{\partial z}-6 H D_{s} \\
\times \frac{\left.\int_{0}^{\infty} D_{p}^{2} f\left(D_{p}\right)\left(\partial C_{s} / \partial r\right)\right|_{r=D_{p} / 2} d D_{p}}{\int_{0}^{\infty} D_{p}^{3} f\left(D_{p}\right) d D_{p}} \\
\frac{\partial C_{s}}{\partial t}=D_{s}\left(\frac{\partial^{2} C_{s}}{\partial r^{2}}+\frac{2}{r} \cdot \frac{\partial C_{s}}{\partial r}\right)
\end{gathered}
$$

where $C$ and $C_{z}$ are the concentrations of a component in the mobile phase and the gel phase, respectively. $D_{t}$

\footnotetext{
+ Analysis of Dispersion Mechanism in Gel Chromatography. Part III. See References 1, 2).
}

is the effective longitudinal dispersion coefficient, $z$ the distance from the top of a column bed, $u$ the actual velocity of a solvent flow, $r$ the radial distance in the particle, $D_{g}$ the gel phase diffusion coefficient, $t$ the time and $H=(1-\varepsilon) / \varepsilon$, where $\varepsilon$ is the void fraction of a column tube. Initial and boundary conditions are given as

$$
\begin{aligned}
& t=0, z \geq 0, C=C_{s}=0 \\
& t>0, r=D_{p} / 2, C_{s}=K C \\
& t>0, r=0, \partial C_{s} / \partial r=0 \\
& 0<t \leq t_{0}, z=0, C=C_{0} \\
& t>t_{0}, z=0, C=0
\end{aligned}
$$

where $K$ is the distribution coefficient of a solute between the gel phase and the space outside the gel particle.

Equations (1) (3) can be easily solved in the Laplace region and for a negligibly small $t_{0}$, the first moment $\mu_{1}^{\prime}$ and the second central moment $\mu_{2}$ of an elution curve $^{1,6,7)}$ are derived as

$$
\begin{gathered}
\mu_{1}^{\prime}=\left(z / u_{0}\right)[\varepsilon+(1-\varepsilon) K] \\
\mu_{2}=(2 z / u)\left[D_{l} / u^{2}(1+H K)^{2}+H K D_{p a}{ }^{2} /\left(60 D_{s}\right)\right]
\end{gathered}
$$

where

$$
D_{p a}=\left[\int_{0}^{\infty} D_{p}^{5} f\left(D_{p}\right) d D_{p} / \int_{0}^{\infty} D_{p}^{3} f\left(D_{p}\right) d D_{p}\right]^{1 / 2}
$$

Equation (4) means that $K$ does not depend on the particle size distribution. From Eqs. (4) and (5), the height equivalent to a theoretical plate (abbreviated as HETP) which expresses column efficiency is obtained $\operatorname{as}^{1,2)}$

$$
\mathrm{HETP}=2 D_{l} / u+H K u D_{p a}{ }^{2} /\left[30 D_{s}(1+H K)^{2}\right]
$$

The first term of the right hand side of Eq. (7) represents the axial dispersion effect in a column. The second term represents the permeation effect in the gel phase and might play a much more important role than the first one. ${ }^{1,2)}$ Thus, in a column containing particles of widely differing sizes, column efficiency might be evaluated as if gels had a uniform size of $D_{p a}$. For instance, when the particle size distribution is represented by the normal (Gauss) distribution with a mean diameter $\bar{D}_{p}$ and standard deviation $\sigma$, i.e., $f\left(D_{p}\right)=$ $\exp \left[-\left(D_{p}-\bar{D}_{p}\right)^{2} / 2 \sigma^{2}\right] /(\sqrt{2 \pi} \sigma), D_{p a}$ is derived from Eq. (6) as

$$
D_{p a}=\bar{D}_{p}\left[\left(1+10 x^{2}+15 x^{4}\right) /\left(1+3 x^{2}\right)\right]^{1 / 2}
$$

where

$$
x=\sigma / \bar{D}_{p}
$$

It is obvious from Eq. (8) that $D_{p a}$ increases with $x$. This means that for the same value of $\bar{D}_{p}$, an apparent particle diameter $D_{p a}$ increases as the size distribution becomes broader, and accordingly column efficiency decreases as seen in Eq. (7). Equation (7) can be rearranged in reduced coordinates, ${ }^{8}$, with $D_{p a}$ as a representative diameter as

$$
h=2 D_{l} / u D_{p a}+H K \nu /\left[30 \gamma(1+H K)^{2}\right]
$$




\section{Table I. Mean Diameters of Original Gels and Sieve Fractions}

The values of diameters and standard deviation are expressed in microns. More than 500 and 200 particles were used in the measurement of particle diameters for original Sephadex gels and the sieve fractions, respectively.

\begin{tabular}{|c|c|c|c|c|c|c|}
\hline Sephadex gel type & $\begin{array}{c}\text { Mode } \\
\text { diameter }\end{array}$ & $\begin{array}{l}\text { Linear } \\
\text { mean } \\
\text { diameter } \\
\left(D_{p 1}\right)^{c}\end{array}$ & $\begin{array}{c}\text { Surface } \\
\text { mean } \\
\text { diameter } \\
\left(D_{p_{2}}\right)^{\mathrm{c}}\end{array}$ & $\begin{array}{l}\text { Volume } \\
\text { mean } \\
\text { diameter } \\
\left(D_{p 3}\right)^{c}\end{array}$ & $D_{x a^{d}}$ & $\begin{array}{c}\text { Standard } \\
\text { deviation }(\sigma)^{e} \\
\left(\sigma / D_{p 1}\right)\end{array}$ \\
\hline G-25 Medium originala & 92.0 & 125.4 & 131.2 & 136.8 & 163.8 & $38.5(0.31)$ \\
\hline G-25 Coarse original ${ }^{a}$ & 170.0 & 216.7 & 233.7 & 241.1 & 280.5 & $60.1(0.28)$ \\
\hline $\mathrm{G}-150$ origina $^{a}$ & 128.3 & 153.9 & 163.9 & 172.8 & 215.0 & $55.4(0.36)$ \\
\hline$G-2580 / 100^{b}$ & 166.0 & 166.8 & 169.4 & 170.7 & 176.9 & $21.1(0.13)$ \\
\hline $\mathrm{G}-2560 / 80^{\circ}$ & 235.0 & 236.8 & 237.6 & 238.7 & 244.6 & $22.0(0.09)$ \\
\hline $\mathrm{G}-15080 / 100^{b}$ & 186.0 & 184.3 & 186.7 & 189.0 & 201.3 & $30.6(0.17)$ \\
\hline
\end{tabular}

a The fine particles remaining in the supernatant when the dry gel was suspended for an appropriate period of time in an aqueous solution were removed by decantation.

${ }^{b}$ The numeral next to $\mathrm{G}-25$ or G-150 represents the sieve fraction of gels, i.e., G-25 80/100 means a fraction at 80 and 100 mesh sizes separated from the original $G-25$ gel.

c The values of $D_{p i}(i=1-3)$ were calulated by equation $D_{p i}=\left(\int_{0}^{\infty} f\left(D_{p}\right) D_{p}{ }^{i} d D_{p} \cdot\right)^{1 / i}$

$d$ The $D_{p a}$ value was calculated by Eq. (6) See the text for details.

- The value of $\sigma$ was calculated by equation $\sigma=\left[\int_{0}^{\infty} f\left(D_{p}\right)\left(D_{p}-D_{p 1}\right)^{2} d D_{p}\right]^{1 / 2}$.

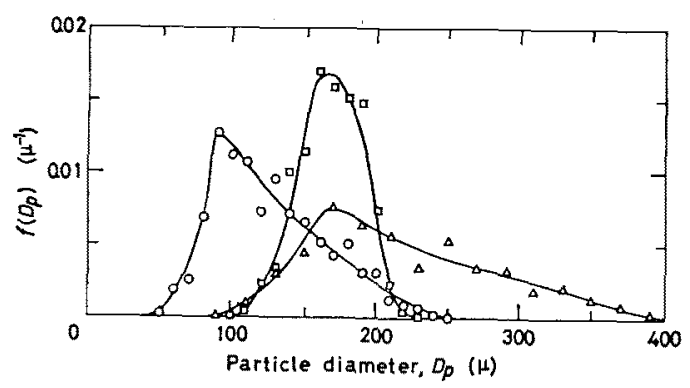

FIG. 1. Typical Particle Size Distribution of Original Sephadex Gels and the Sieve Fraction.

$\bigcirc$, original Sephadex G-25 medium; $\triangle-\triangle$, original Sephadex G-25 Coarse; $\square-\square$, sieve fraction at $80 \sim 100$ mesh sizes of G-25 Medium. See the footnote in Table I for details.

where

$h=\operatorname{HETP} / D_{p a}$

$\nu=u D_{p a} / D_{m}\left(D_{m}:\right.$ the molecular diffusion coefficient $)$

$\gamma=D_{\mathrm{s}} / D_{m}$

Figure 1 shows the particle size distribution observed for the original Sephadex G-25 Medium, G-25 Coarse and the sieve fraction at $80 \sim 100$ mesh sizes of G-25 Medium gel. Original gels showed broad and asymmetrical distribution curves and could be expressed neither by the normal nor by the log-normal distribution function. On the other hand, the sieve fraction showed a relatively narrow and symmetrical curve. In
Table I are summarized several mean diameters including the one defined by Eq. (6) and standard deviation from the linear mean diameter evaluated for the gels used in the experiment. As seen in Table $I, \sigma / D_{p 1}$ is larger for the original gels than that for the sieve fraction, and $D_{p a}$ becomes noticeably larger than surface mean and volume mean diameters as well as a linear mean diameter. However, the sieve fractions had relatively small values of $\sigma / D_{p 1}$, and only a small difference was observed among the mean diameters shown in Table I.

To examine the efficiency of these gel columns, the HETP values were calculated from the experimental data of $\mu_{1}^{\prime}$ and $\mu_{2}$ of the elution curve, in which $\mathrm{NaCl}$ and myoglobin were used as samples. In Fig. 2, the HETP values are plotted against the flow velocity in the reduced coordinates. As seen in the figure, all the experimental data in the same gel types seem to gather on each single line regardless of the particle size distribution. This means the validity of Eq. (9). Thus, it might be reasonable to adopt $D_{p a}$ as a representative diameter instead of the other mean diameters shown in Table I when one is to evaluate the efficiency of columns containing gels of widely distributed particle sizes. It should be emphasized that the experimental data could not be expressed by a single line when any other diameter such as a linear mean diameter was used as a representative diameter.

It is easily found from Eq. (9) that the slope of the line in Fig. 2 is expressed by $H K /\left[30 \gamma(1+H K)^{2}\right]$. The values of $\varepsilon$ and $K$ were slightly varied with particle size distribution, and the mean values were evaluated to be 


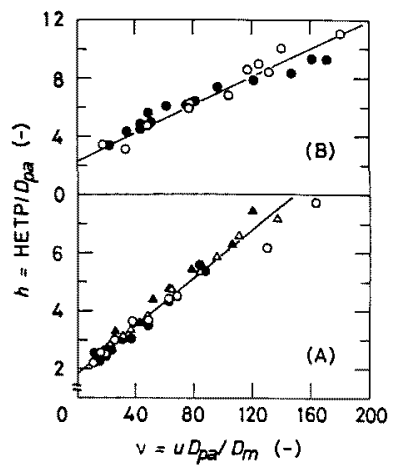

FIG. 2. Experimental Relations between Reduced HETP and Reduced Flow Velocity for Original Sephadex Gels and the Sieve Fractions.

The temperature was controlled at $20^{\circ} \pm 0.5^{\circ} \mathrm{C}$ by circulating water through a jacket. As the values of $D_{m}$, $1.35 \times 10^{-5} \mathrm{~cm}^{2} / \mathrm{sec}^{0)}$ and $1.13 \times 10^{-8} \mathrm{~cm}^{2} / \mathrm{sec}^{10)}$ were used for $\mathrm{NaCl}$ and myoglobin, respectively.

(A) Bed length, $20 \mathrm{~cm}$; sample, $1 \mathrm{M} \mathrm{NaCl}$; eluant, $1 / 100 \mathrm{M} \mathrm{NaCl}$; $O$, original $\mathrm{G}-25$ Medium column; $\triangle$, original G-25 Coarse column; , G-25 80/100 column (sieve fraction at $80 \sim 100$ mesh sizes); $\boldsymbol{\Delta}, \mathrm{G}-2560 / 80$ column.

(B) Bed length, $13 \mathrm{~cm}$; sample, $1 \%$ myoglobin; eluant, $1 / 10 \mathrm{M}$ Tris- $\mathrm{HCl}$ buffer $\mathrm{pH} 7.5 ; 0$, original G-150 column; , G-150 80/100 column.

0.41 and 0.73 for $\mathrm{NaCl}$ with $\mathrm{G}-25$ columns and 0.42 and 0.69 for myoglobin with $\mathrm{G}-150$ columns. By using these values of $\varepsilon$ and $K$ and slopes shown in Fig. 2, 0.21 and 0.18 were obtained as the $\gamma$ values for $\mathrm{NaCl}$ with G-25 columns and myoglobin with G-150 columns.
The $\gamma$ values are still much smaller than those obtained for a single gel bead, i.e., 0.36 for $\mathrm{NaCl}^{1)}$ and 0.38 for myoglobin. ${ }^{2)}$ This might imply the apparent increase of the particle size possibly caused by channelling in a column as mentioned in previous papers. ${ }^{1,2)}$

The effect of particle size distribution on the channelling might be negligible, since the experimental relations between $h$ and $\nu$ were expressed by single lines regardless of the paricle size distribution as shown in Fig. 2. However, further investigations must be required on this problem in connection with packing heterogeneity.

\section{REFERENCES}

1) K. Nakanishi, S. Yamamoto, R. Matsuno and T. Kamikubo, Agric. Biol. Chem., 41, 1465 (1977).

2) S. Yamamoto, K. Nakanishi, R. Matsuno and T. Kamikubo, 42, 963 (1978).

3) H. Determann and J. E. Brewer, "Chromatography," 3rd ed., ed. by E. Heftmann, Van Nostrand Reinhold Co., New York, 1975, p. 377.

4) A. J. de Vries, M. LePage, R. Beau and C. L. Guillemin, Anal. Chem., 39, 935 (1967).

5) M. Kubin, Collect. Czech. Chem. Commun., 30, 1104 (1965).

6) F. Billmeyer, Jr., G. W. Johnson and R. N. Kelley, J. Chromatogr., 34, 316, 322 (1968).

7) M. Suzuki, J. Chem. Eng. Japan, 7, 262 (1974).

8) J. C. Giddings, Anal. Chem., 35, 1338 (1963).

9) T. Takamatsu, M. Hiraoka, T. Tanaka, Y. Inoue and A. Osugi, Kagaku Kogaku, 28, 451 (1964).

10) T. C. Laurent and J. Killander, J. Chromatogr., 14, 317 (1964). 